\title{
Qualidade de vida, ansiedade e depressão em estudantes de Odontologia na pandemia da COVID-19 e fatores relacionados
}

\author{
Quality of life, anxiety and depression in Dentistry students in the COVID-19 pandemic and related \\ Calidad de vida, ansiedad y depresión en estudiantes de Odontología en la pandemia del COVID-19 \\ y factores afines
}

Recebido: 22/06/2021 | Revisado: 28/06/2021 | Aceito: 30/06/2021 | Publicado: 13/07/2021

\author{
Thauany Vasconcelos Soares da Silva \\ ORCID: https://orcid.org/0000-0002-6831-9224 \\ Universidade Federal da Paraíba, Brasil \\ E-mail: vasconcelosthauany@gmail.com \\ Louandrys Montenegro Vieira \\ ORCID: https://orcid.org/0000-0002-7367-2954 \\ Centro Universitário de João Pessoa, Brasil \\ E-mail: louandrys@gmail.com \\ Andreia Medeiros Rodrigues Cardoso \\ ORCID: https://orcid.org/0000-0002-2682-1048 \\ Centro Universitário de João Pessoa, Brasil \\ E-mail: andreia.medeiros@unipe.edu.br \\ Rosa Virginia Dutra de Oliveira \\ ORCID: https://orcid.org/0000-0003-3842-1436 \\ Centro Universitário de João Pessoa, Brasil \\ E-mail: drarosadutra@gmail.com
}

\begin{abstract}
Resumo
Objetivo: Avaliar o impacto do isolamento social na qualidade de vida de estudantes de odontologia. Metodologia: Utilizou-se formulários Google abordando aspectos sociodemográficos; avaliação da qualidade de vida (WHOQOLbreve) e escala de ansiedade, depressão e estresse (EADS-21).Dados foram analisados com testes Mann Whitney e Kruskal-Wallis; além de Correlação de Pearson para verificar relação entre escores de WHOQOL-breve e EADS$21(\alpha=0.05)$. Resultados e Discussão: Participaram 249 estudantes, sendo 80,3\% mulheres, entre 18 e 23 anos (70,7\%), que estavam em capitais $(57,8 \%)$, com familiares ou parentes $(95,2 \%)$. Observou-se níveis normais de estresse $(46,2 \%)$, ansiedade $(39,4 \%)$ e depressão $(41,8 \%)$. Houve prejuízo nos domínios físico $(46,42 \pm 13,12)$ e psicológico $(62,50 \pm 18,75)$ da qualidade de vida. Mulheres e estudantes mais jovens apresentaram níveis significativamente maiores de estresse, ansiedade e depressão. Mulheres foram mais prejudicadas nos domínios físico, psicológico e ambiental; estudantes mais jovens, no domínio psicológico. Observou-se prejuízo significativo no domínio físico aqueles que estiveram em cidades interioranas e nos domínios psicológico e social daqueles que estiveram sozinhos. Houve correlação positiva entre as variáveis estresse, ansiedade e depressão; além de correlação negativa entre essas variáveis e os domínios de qualidade de vida, sendo maior entre depressão e o domínio psicológico. Conclusão: Os achados evidenciaram que estudantes mais jovens, do sexo feminino, que estiveram sozinhos e em cidades do interior apresentaram pior qualidade de vida.
\end{abstract}

Palavras-chave: COVID-19; Estudantes de odontologia; Ansiedade; Ensino.

\begin{abstract}
Objective: To assess the impact of social isolation on the quality of life of dental students. Methodology: used Google forms addressing sociodemographic aspects; assessment of quality of life (WHOQOL-brief) and scale of anxiety, depression and stress (EADS-21). Data analyzed with Mann Whitney and Kruskal-Wallis tests; and Pearson's Correlation to verify the relationship between WHOQOL-brief and EADS-21 scores $(\alpha=0.05)$. Results and Discussion: 249 students participated, $80.3 \%$ being women, between 18 and 23 years old (70.7\%), who were in capitals $(57.8 \%)$, with relatives or relatives $(95.2 \%)$. Observe norms of stress $(46.2 \%)$, anxiety $(39.4 \%)$ and depression $(41.8 \%)$. There was prejudiced us physical $(46.42 \pm 13.12)$ and psychological $(62.50 \pm 18.75)$ domains of quality of life. Young women and students will experience significantly higher levels of stress, anxiety and depression. Women were most harmed us physical, psychological and environmental domains; Younger students, no psychological domain. It was observed that a significant non-physical domain prejudice was those who estimate internal cities in our psychological and social domain, those who estimate alone. There is a positive correlation between the various stresses, anxieties and depression; There is also a negative correlation between these various domains of quality of life, being higher between depression and the psychological domain. Conclusion: You will
\end{abstract}


show that younger students, of feminine sex, that they estimate their inner situations and inner cities, they will show higher quality of life.

Keywords: COVID-19; Students, dental; Anxiety; Teaching.

\begin{abstract}
Resumen
Objetivo: Evaluar el impacto del aislamiento social en la calidad de vida de los estudiantes de odontología. Metodología: se utilizaron formularios de Google que abordan aspectos sociodemográficos; evaluación de la calidad de vida (WHOQOL-brief) y escala de ansiedad, depresión y estrés (EADS-21) Los datos se analizaron mediante las pruebas de Mann Whitney y Kruskal-Wallis; además de la correlación de Pearson para verificar la relación entre las puntuaciones WHOQOL-brief y EADS-21 $(\alpha=0,05)$. Resultados y Discusión: participaron 249 estudiantes, 80,3\% mujeres, entre 18 y 23 años (70,7\%), que se encontraban en capitales $(57,8 \%)$, con familiares o parientes $(95,2 \%)$. Se observaron niveles normales de estrés $(46,2 \%)$, ansiedad $(39,4 \%)$ y depresión $(41,8 \%)$. Hubo una pérdida en los dominios físico $(46,42 \pm 13,12)$ y psicológico $(62,50 \pm 18,75)$ de la calidad de vida. Las mujeres y estudiantes más jóvenes tenían niveles significativamente más altos de estrés, ansiedad y depresión. Las mujeres sufrieron más daños en los ámbitos físico, psicológico y ambiental; estudiantes más jóvenes, en el dominio psicológico. Hubo una pérdida significativa en el dominio físico de quienes habían estado en el centro de las ciudades y en los dominios psicológicos y sociales de quienes estaban solos. Hubo una correlación positiva entre las variables estrés, ansiedad y depresión; además de una correlación negativa entre estas variables y los dominios de la calidad de vida, siendo mayor entre la depresión y el dominio psicológico. Conclusión: Estudiantes más jóvenes que estaban solas y en el centro de las ciudades tenían una peor calidad de vida.
\end{abstract}

Palabras clave: COVID-19; Estudiantes de odontología; Ansiedad; Ensenãnza.

\title{
1. Introdução
}

A atual emergência de saúde pública é uma doença infecciosa causada por um vírus - o coronavírus. Desde o início de fevereiro, a Organização Mundial da Saúde (OMS) passou a denominar oficialmente a doença de COVID-19. O primeiro caso foi relatado na China, em Wuhan, província de Hubei e em pouco tempo se propagou para outros países (Guo et al., 2020; Moreira \& Tonon, 2021). Teorias sugerem que a doença é semelhante a síndrome respiratória aguda grave causada pelo coronavírus tipo 2 (SARS-CoV-2), todavia, as características relacionadas ao novo coronavírus merecem ser ainda mais investigadas (Spagnuolo et al., 2020).

Na sintomatologia, é possível notar relatos de febre, tosse, perda da sensibilidade gustativa e olfatória, diarréia, e em casos mais graves, doença respiratória aguda, pneumonia, insuficiência renal, podendo até mesmo levar o indivíduo a morte. Normalmente os sintomas são leves, porém, as estatísticas mostram que pacientes com idade avançada ou comprometimento sistêmico apresentam maior risco de morte. O diagnóstico é realizado por meio da coleta de amostras da nasofaringe, orofaringe e sangue, submetidos a testes laboratoriais (Sabino-silva; Jardim; Siqueira, 2020).

O contágio ocorre por meio de gotículas de saliva, espirros e tosse, podendo ser ponderada também a transmissão pelo sangue (Guo et al., 2020; Bezerra et al., 2020; Cardoso et al., 2021). Assim, a produção de aerossóis contaminados por saliva e/ou sangue que ocorre durante a realização de determinados procedimentos odontológicos coloca os profissionais e estudantes de Odontologia no foco da transmissão do COVID-19 (Guo et al., 2020). Ao prestar atendimento clínico a pessoas doentes ou que ainda não apresentam diagnóstico (assintomáticas), profissionais e estudantes correm o risco não só de se contaminar, como também contaminar sua equipe e/ou outros pacientes (Bezerra et al., 2020; Schmidt et al., 2020). Para evitar essa contaminação, é de suma importância que esses profissionais e estudantes utilizem medidas de proteção adequadas (Sabino-Silva; Jardim; Siqueira, 2020).

Devido à facilidade de transmissão do COVID-19, vários países adotaram medidas de isolamento social, com o intuito de não sobrecarregar os sistemas de saúde. Essa fase de reclusão pode trazer efeitos negativos para a saúde mental, por isso, a psicologia procura discutir o impacto desses eventos em relação as respostas cerebrais dos indivíduos. Para muitos, a situação da pandemia pode gerar pânico, confusão maiores índices de ansiedade e depressão, já que é uma situação nova e que modifica a rotina dos indivíduos (Mailloy-Diniz et al., 2020). Além disso, o isolamento social pode despertar sentimentos de angústia, insegurança e medo, que podem se prolongar até mesmo depois do controle da disseminação do vírus (Mailloy-Diniz et al., 
2020; Hossain et al., 2020).

O medo de ser infectado por um vírus de fácil disseminação, que ocasiona uma doença ainda sem tratamento eficaz definido e que tem causado tantos óbitos em todo o mundo, pode afetar sobremaneira o bem-estar psicológico das pessoas Hossain et al., 2020; Carvalho et al., 2020).

Preocupações com as perdas financeiras também podem acarretar prejuízos ao bem-estar psicológico (Shojae; Masoum, 2020). Todos esses fatores remetem à relevância de se pesquisar a respeito da saúde mental e bem-estar de jovens estudantes em fase de formação profissional submetidos a esse período de pandemia de isolamento.

Assim, o objetivo do presente estudo foi avaliar o impacto da pandemia da COVID-19 na qualidade de vida; caracterizar o perfil sócio demográfico; avaliar a qualidade de vida geral;verificar a presença de sintomas de ansiedade, estresse e depressão; avaliar se existe relação entre qualidade de vida e sintomas de ansiedade, estresse e depressão;correlacionar as variáveis sócio demográficas a qualidade de vida e sintomas de ansiedade, estresse e depressão de Odontologia de uma instituição de João Pessoa, localizado no nordeste do Brasil.

\section{Metodologia}

Esta pesquisa é caracterizada como um estudo quantitativo, exploratório, descritivo, analítico e transversal. Uma vez que envolveu seres humanos, a pesquisa só foi desenvolvida após a aprovação do Comitê de Ética em Pesquisa (CEP), do Centro Universitário de João Pessoa (UNIPÊ), sob número CAAE33359820.8.0000.5176 e conforme Parecer Consubstanciado 4.102.491.

O universo foi constituído por 678 estudantes matriculados do $1^{\circ}$ ao $10^{\circ}$ períodos do curso de Odontologia de uma instituição privada de João Pessoa, capital da Paraíba e no nordeste do Brasil. Após cálculo amostral, determinou-se que a amostra seria composta por 246 estudantes (com nível de confiança de 95\% e margem de erro de 5\%). Os participantes foram selecionados de forma não probabilística. O link para acesso ao questionário online foi enviado a todos os alunos que compunham o universo do estudo, encerrando-se a coleta ao atingir o número determinado no cálculo amostral. Considerou-se os seguintes critérios de inclusão: estar regularmente matriculado no curso; ser maior de 18 anos no momento da realização da pesquisa.

Utilizou-se a ferramenta Formulários Google da Microsoft para abordagem dos estudantes. O link contendo o formulário ficou disponível durante do período de 01 agosto de 2020 até 30 de setembro de 2020.0 link de acesso ao questionário online foi encaminhado aos alunos, através da Coordenação do Curso.

O instrumento para coleta de dados foi composto por: um questionário abordando aspectos sócio demográficos, o Questionário de Avaliação da Qualidade de vida WHOQOL-breve, traduzido para o português e validado por Fleck et al. (1999) e a Escala de ansiedade, depressão e estresse (EADS-21) de Lovibond e Lovibond (1995), traduzido para o português e validado por Pais Ribeiro, Honrado e Leal (2004). O questionário com dados sociodemográficos abordou questões relacionadas à sexo, idade, semestre que estava cursando, onde e com quem o aluno esteve durante o período de isolamento social.

O WHOQOL-breve é um questionário autoaplicável, com 26 questões que apresenta quatro domínios da qualidade de vida: físico, psicológico, meio ambiente e relações sociais. Para cada aspecto da qualidade de vida expresso no questionário WHOQOL/breve, o sujeito pode apresentar sua resposta por meio de escores que variam de um a cinco, sendo a condição pior no escore um e a melhor, no cinco. Os resultados dos domínios apresentam valores entre zero e cem, sendo piores os mais próximos de zero e melhores, os mais próximos de cem.A Escala de ansiedade, depressão e estresse (EADS-21) de Lovibond e Lovibond (1995), foi traduzida para o português e validada por Pais Ribeiro, Honrado e Leal (2004). Essa escala pretende avaliar os sintomas associados à ansiedade, depressão e estresse em jovens adultos. É constituída por 21 itens, agrupados em 
três subescalas (Ansiedade, Depressão e Estresse), constituídas por 7 itens cada.

Todos os itens são avaliados através de uma escala de resposta de tipo Likert, de 4 pontos, que reenviam para a severidade e frequência dos sintomas experimentados durante o período de isolamento social (0 - "não se aplicou nada a mim", 1 - "aplicou-se a mim algumas vezes", 2 - "aplicou-se a mim muitas vezes", e 3 - "aplicou-se a mim a maior parte das vezes"). A cotação é dada pela soma dos resultados dos 7 itens, obtendo-se uma nota para cada subescala com um resultado mínimo de 0 e máximo de 21. Desta forma, notas mais elevadas correspondem a estados afetivos mais negativos.

A estatística descritiva foi realizada para caracterizar a amostra. Testes de normalidade foram realizados para analisar a distribuição dos dados. Os dados dos escores dos questionários estudados foram comparados de acordo com a condições demográficas e de isolamento social, por meio dos Testes não paramétricos de Mann Whitney e Kruskal Wallis. A Correlação de Pearson foi realizada para verificar a relação dos escores dos questionários de Qualidade de vida e do EADS-21. Todos os testes foram realizados no software Statistical Package for the Social Sciences (SPSS para Windows, versão 20.0), considerando o nível de significância de 5\%.

\section{Resultados}

Um total de 249 participantes compuseram a amostra, sendo $80,3 \%$ do sexo feminino e $43 \%$ na faixa etária de 21 e 23 anos (Tabela 1). Durante o período isolamento social observou-se que 57,8\% dos estudantes estavam em João Pessoa ou outras capitais e a maior parte deles, com familiares ou parentes $(95,2 \%), 4 \%$ sozinhos e $0,8 \%$ com amigos. 
Tabela 1: Dados sociodemográficos.

\begin{tabular}{|c|c|c|}
\hline Variáveis & $\mathbf{N}$ & $\%$ \\
\hline \multicolumn{3}{|l|}{ Sexo } \\
\hline Feminino & 200 & 80,3 \\
\hline Masculino & 49 & 19,7 \\
\hline \multicolumn{3}{|l|}{ Faixa etária } \\
\hline De 18 a 20 anos & 69 & 27,7 \\
\hline De 21 a 23 anos & 107 & 43 \\
\hline De 24 a 26 anos & 31 & 12,4 \\
\hline De 27 a 29 anos & 13 & 5,2 \\
\hline Mais de 30 anos & 29 & 11,6 \\
\hline \multicolumn{3}{|l|}{ Semestre que está cursando } \\
\hline $1^{\mathrm{o}}$ semestre & 18 & 7,2 \\
\hline $2^{\circ}$ semestre & 7 & 2,8 \\
\hline $3^{\circ}$ semestre & 18 & 7,2 \\
\hline $4^{\mathrm{o}}$ semestre & 11 & 4,4 \\
\hline $5^{\circ}$ semestre & 28 & 11,2 \\
\hline $6^{\circ}$ semestre & 12 & 4,8 \\
\hline $7^{\circ}$ semestre & 34 & 13,7 \\
\hline $8^{\circ}$ semestre & 19 & 7,6 \\
\hline $9^{\circ}$ semestre & 54 & 21,7 \\
\hline $10^{\circ}$ semestre & 48 & 19,3 \\
\hline \multicolumn{3}{|c|}{ Onde estava durante o período de isolamento social } \\
\hline Em João Pessoa ou outras capitais & 144 & 57,8 \\
\hline Em cidades do interior & 105 & 42,2 \\
\hline \multicolumn{3}{|c|}{ Com quem esteve durante o período do isolamento } \\
\hline Com familiares ou parentes & 237 & 95,2 \\
\hline Com amigos & 2 & 0,8 \\
\hline Sozinho & 10 & 4 \\
\hline
\end{tabular}

Fonte: Pesquisa própria (2021).

Na Tabela 2 tem-se a média dos escores de qualidade de vida dos universitários, por domínio. Observou-se que os domínios físico e psicológico apresentaram as menores médias, indicando que os estudantes se sentiram mais comprometidos nesses domínios.

Tabela 2: Média dos escores de qualidade de vida dos universitários do curso de Odontologia, de acordo com os domínios.

\begin{tabular}{cc}
\hline Domínios de qualidade de vida & Média \pm Desvio Padrão \\
\hline Físico & $46,42 \pm 13,12$ \\
Psicológico & $62,50 \pm 18,75$ \\
Social & $66,66 \pm 21,93$ \\
Ambiental & $65,62 \pm 16,63$ \\
\hline
\end{tabular}

Fonte: Pesquisa própria (2021). 
Na Tabela 3 tem-se a avaliação da presença de sintomas de estresse, ansiedade e depressão medido pela Escala EADS-21. A maior parte dos estudantes apresentaram níveis considerados normais de estresse, ansiedade e depressão.

Tabela 3: Ansiedade, Depressão e Estresse dos estudantes.

\begin{tabular}{|c|c|c|c|c|c|c|c|c|c|c|}
\hline \multirow[b]{3}{*}{ Variáveis } & \multicolumn{10}{|c|}{ Classificação EADS } \\
\hline & \multicolumn{2}{|c|}{ Normal } & \multicolumn{2}{|c|}{ Leve } & \multicolumn{2}{|c|}{ Moderada } & \multicolumn{2}{|c|}{ Severa } & \multicolumn{2}{|c|}{$\begin{array}{c}\text { Extremamente } \\
\text { severa }\end{array}$} \\
\hline & $\mathrm{N}$ & $\%$ & $\mathrm{~N}$ & $\%$ & $\mathrm{~N}$ & $\%$ & $\mathrm{~N}$ & $\%$ & $\mathrm{~N}$ & $\%$ \\
\hline Estresse & 115 & 46,2 & 29 & 11,6 & 37 & 14,9 & 29 & 11,6 & 3 & 1,2 \\
\hline Ansiedade & 98 & 39,4 & 25 & 10 & 53 & 21,3 & 15 & 6 & 34 & 13,7 \\
\hline Depressão & 104 & 41,8 & 26 & 10,4 & 50 & 20,1 & 18 & 7,2 & 11 & 4,4 \\
\hline
\end{tabular}

Fonte: Pesquisa própria (2021).

A Tabela 4 apresenta a comparação dos escores dos domínios de qualidade de vida e do EADS-21 de acordo com o sexo. Observou-se que as mulheres foram mais comprometidas nos domínios físico, psicológico e ambiental da qualidade de vida, com diferença significativa, além de níveis significativamente maiores de estresse, ansiedade e depressão.

Tabela 4: Comparação dos escores dos domínios de qualidade de vida e do EADS-21 de acordo com o sexo.

\begin{tabular}{ccc|c}
\hline & \multicolumn{2}{c|}{ Mediana (Q25-75) } & Valor de $p$ \\
Domínios de qualidade de & Feminino & Masculino & \\
vida & $46,42(39,28-57,14)$ & $57,14(46,42-64,28)$ & 0,001 \\
\hline Físico & $58,33(50,00-70,83)$ & $75,00(60,41-83,33)$ & 0,000 \\
Psicológico & $66,66(50,00-75,00)$ & $66,66(58,33-79,16)$ & 0,598 \\
Social & $65,62(53,12-80,46$ & $75,00(62,50-89,06)$ & 0,017 \\
Ambiental & & & 0,005 \\
Variáveis EADS & $14,00(10,00-23,50)$ & $10,00(7,00-18,00)$ & 0,034 \\
Estresse & $8,00(2,00-14,00)$ & $6,00(0,00-12,00)$ & 0,002 \\
\hline Ansiedade & $10,00(6,00-18,00)$ & $6,00(4,00-12,00)$ & \\
\hline
\end{tabular}

Teste de Mann-Whitney U (p<0,05). Fonte: Pesquisa própria (2021).

A Tabela 5 apresenta a comparação dos escores dos domínios de qualidade de vida e do EADS-21 de acordo com a faixa etária. Observou-se que os estudantes mais jovens foram significativamente mais prejudicados no domínio psicológico da qualidade de vida e apresentaram níveis significativamente maiores de estresse, ansiedade e depressão. 
Tabela 5: Comparação dos escores dos domínios de qualidade de vida e do EADS-21 de acordo com a faixa etária.

\begin{tabular}{ccc|c}
\hline & \multicolumn{2}{c|}{ Mediana (Q25-75) } & \multirow{2}{*}{ Valor de $\mathrm{p}$} \\
Domínios de qualidade de & De 18 a 26 anos & Mais de 27 anos & \\
vida & & $50,00(42,85-57,14)$ & 0,412 \\
\hline Físico & $46,42(39,28-57,14)$ & $62,50(54,16-79,16)$ & 0,034 \\
Psicológico & $62,50(50,00-70,83)$ & $66,66(50,00-75,00)$ & 0,959 \\
Social & $66,66(50,00-75,00)$ & $65,62(48,43-81,25)$ & 0,755 \\
Ambiental & $67,18(59,37-81,25)$ & & 0,007 \\
\hline Variáveis EADS & $16,00(10,00-23,50)$ & $12,00(12,00-18,00)$ & 0,004 \\
Estresse & $8,00(4,00-14,00)$ & $4,00(4,00-12,00)$ & 0,000 \\
\hline
\end{tabular}

Teste de Mann-Whitney U (p<0,05). Fonte: Pesquisa própria (2021).

A Tabela 6 apresenta a comparação dos escores dos domínios de qualidade de vida e do EADS-21 de acordo com o local onde os estudantes estiveram durante o período de isolamento social. Observou-se que os estudantes que estiveram em cidades do interior foram significativamente mais prejudicados no domínio físico da qualidade de vida, entretanto não houve diferença significativa nos níveis de estresse, ansiedade e depressão.

Tabela 6: Comparação dos escores dos domínios de qualidade de vida e do EADS-21 de acordo com o local onde os estudantes estiveram durante o período de isolamento social.

\begin{tabular}{ccc|c}
\hline & \multicolumn{2}{c|}{ Mediana (Q25-75) } & \multirow{2}{*}{$\begin{array}{c}\text { Valor de } p \\
\text { vomínios de qualidade de }\end{array}$} \\
$\begin{array}{cccc}\text { João Pessoa ou outras } \\
\text { capitais }\end{array}$ & Cidades do interior & \\
\hline Físico & $50,00(40,17-60,71)$ & $46,42(35,71-53,57)$ & 0,034 \\
Psicológico & $62,50(50,00-75,00)$ & $58,33(50,00-75,00)$ & 0,376 \\
Social & $66,66(50,00-75,00)$ & $66,66(50,00-75,00)$ & 0,939 \\
Ambiental & $68,75(59,37-81,25)$ & $65,62(54,68-75,00)$ & 0,074 \\
\hline Variáveis EADS & $14,00(10,00-22,00)$ & $12,00(12,00-18,00)$ & 0,724 \\
\hline Estresse & $8,00(2,00-14,00)$ & $4,00(4,00-12,00)$ & 0,172 \\
Ansiedade & $10,00(6,00-16,00)$ & $6,00(6,00-12,00)$ & 0,681 \\
\hline
\end{tabular}

Teste de Mann-Whitney U (p<0,05). Fonte: Pesquisa própria (2021).

A Tabela 7 apresenta a comparação dos escores dos domínios de qualidade de vida e do EADS-21 de acordo com a convivência durante o período do isolamento social. Observou-se que as estudantes que estiveram sozinhos durante o período de isolamento social foram significativamente mais prejudicados nos domínios psicológico e social da qualidade de vida. Não houve diferença significativa nos níveis de estresse, ansiedade e depressão. 
Tabela 7: Comparação dos escores dos domínios de qualidade de vida e do EADS-21 de acordo com a convivência durante o período do isolamento social.

\begin{tabular}{|c|c|c|c|c|}
\hline \multirow[b]{2}{*}{$\begin{array}{c}\text { Domínios de } \\
\text { qualidade de } \\
\text { vida }\end{array}$} & \multicolumn{4}{|c|}{ Mediana (Q25-75) } \\
\hline & Familiares ou parentes & Amigos & Sozinho & Valor de $\mathrm{p}$ \\
\hline Físico & $50,00(39,28-57,14)$ & $44,64(42,85)$ & $41,78(39,28-48,21)$ & 0,225 \\
\hline Psicológico & $62,50(50,00-75,00)$ & $52,08(33,33)$ & $41,25(8,33-62,50)$ & 0,027 \\
\hline Social & $66,66(50,00-75,00)$ & $62,50(41,66)$ & $37,50(14,58-77,08)$ & 0,042 \\
\hline Ambiental & $65,62(59,37-81,25)$ & $62,50(50,00)$ & $55,31(28,12-71,87)$ & 0,202 \\
\hline \multicolumn{5}{|l|}{ Variáveis } \\
\hline \multicolumn{5}{|l|}{ EADS } \\
\hline Estresse & $14,00(10,00-22,00)$ & $12,00(12,00-18,00)$ & $23,00(12,00-28,00)$ & 0,366 \\
\hline Ansiedade & $8,00(2,00-14,00)$ & $4,00(4,00-12,00)$ & $20,00(6,00-28,00)$ & 0,153 \\
\hline Depressão & $10,00(6,00-16,00)$ & $6,00(6,00-12,00)$ & $20,00(6,00-28,00)$ & 0,302 \\
\hline
\end{tabular}

Teste de Kruskal Wallis (p<0,05). Fonte: Pesquisa própria (2021).

Na Tabela 8 tem-se a correlação entre estresse, ansiedade e depressão. Houve correlação positiva significativa entre estresse e ansiedade; entre estresse e depressão; e entre depressão e ansiedade.

Tabela 8: Correlação entre estresse, ansiedade e depressão.

\begin{tabular}{lccc}
\hline Variáveis EADS-21 & Estresse & Ansiedade & Depressão \\
\hline Estresse & & $0,794 * *$ & $0,782 * *$ \\
Ansiedade & $0,794 * *$ & & $0,752 * *$ \\
Depressão & $0,782 * *$ & $0,752 * *$ & \\
\hline
\end{tabular}

Correlação de Pearson $(* * p<0,05)$. Fonte: Pesquisa própria (2021).

Na Tabela 9 tem-se a correlação entre estresse, ansiedade e depressão e os domínios de qualidade de vida.Houve forte correlação negativa entre depressão e o domínio psicológico, o que significa que quanto mais intensos os sintomas de depressão, pior a qualidade de vida - domínio psicológico ( $\mathrm{r}=-0,721 * *)$. Também houve correlação negativa significativa entre estresse e ansiedade com domínio psicológico (respectivamente $\mathrm{r}=-0,599 * *$ e $\mathrm{r}=-0,501 * *$ ) as variáveis estresse, ansiedade e depressão e todos os domínios de qualidade de vida. 
Tabela 9: Correlação entre estresse, ansiedade e depressão e os domínios de qualidade de vida.

\section{Variáveis EADS-21}

\begin{tabular}{cccc}
$\begin{array}{c}\text { Domínios de qualidade de } \\
\text { vida }\end{array}$ & Estresse & Ansiedade & Depressão \\
\hline Físico & $-0,284^{* *}$ & $-0,194^{* *}$ & $-0,341^{* *}$ \\
Psicológico & $-0,599^{* *}$ & $-0,501 * *$ & $-0,721^{* *}$ \\
Social & $-0,243^{* *}$ & $-0,266^{* *}$ & $-0,404^{* *}$ \\
Ambiental & $-0,193^{* *}$ & $-0,176^{* *}$ & $-0,281^{* *}$ \\
\hline
\end{tabular}

Correlação de Pearson (**p<0,01). Fonte: Pesquisa própria (2021).

\section{Discussão}

Os resultados do presente estudo mostraram um real impacto do isolamento social durante a pandemia da COVID-19 na qualidade de vida dos estudantes de Odontologia. De um modo geral, observou-se que a maior parte dos estudantes apresentaram níveis considerados normais de estresse, ansiedade e depressão, porém houve prejuízo nos domínios físico e psicológico da qualidade de vida (Tabelas 2 e 3 )

Contrariamente a esses resultados, observou-se níveis significativamente mais elevados de depressão, ansiedade e estresse em estudantes universitários brasileiros de diversos cursos no período pandêmico, quando comparado a períodos anteriores/normais (Oxfam Brasil, 2020). Observaram impacto psicológico de moderado a grave entre os estudantes chineses (Wang et al., 2020). As epidemias podem levar ao surgimento de sintomas psicológicos em pessoas que antes não os tinham, como também agravar sintomas em pessoas com condições pré-existentes (Hossain et al., 2020).

Sugere-se que a percepção de prejuízo no domínio físico pode estar relacionada ao maior tempo em casa, decorrente da necessidade do isolamento social (Poulsen et al., 2019); a falta de atividade física (Mailloy-Diniz et al., 2020) e o tempo dedicado as aulas remotas (Iyer; Aziz; Ojcus, 2020).

Já o prejuízo relacionado ao domínio psicológico pode ter ocorrido devido às mudanças repentinas na rotina, medo de adoecer, frustração, tédio, informações controversas, dificuldade de aquisição de gêneros de primeira necessidade, perdas financeiras e estigma (Wang et al., 2020). De fato, durante a fase inicial da pandemia, observou-se que mais da metade da população chinesa apresentou não só medo de adoecer, mas também sintomas de depressão, ansiedade e estresse (Gonçalves et al., 2018). No caso da odontologia, além dos estudantes estarem enfrentando todos os desafios associados ao ensino remoto, ainda existe a preocupação de ser infectado pelo vírus durante a prática clínica, devido à facilidade de disseminação por meio de aerossóis (Mailloy-Diniz et al., 2020). Todos esses fatores, juntamente com preocupações relacionadas as perdas financeiras também podem acarretar prejuízos ao bem-estar psicológico (Maia \& Dias. 2020). Houve prejuízo significativo das mulheres nos domínios físico, psicológico e ambiental da qualidade de vida, além de níveis significativamente maiores de estresse, ansiedade e depressão (Tabela 4), o que condiz com outros estudos epidemiológicos com estudantes da faculdade UNESP (Araraquara) os quais relatam que a prevalência de problemas relacionados à saúde mental em mulheres foi duas vezes maior, em comparação aos homens (Barros et al., 2020; Gonzales et al., 2020). Um estudo que buscou avaliar os segmentos demográficos mais afetados durante a pandemia do COVID-19, trouxe resultados que se assemelham a presente pesquisa, onde as mulheres graduandas da UNESP (Araraquara) apresentaram maior tristeza, nervosismo e alterações durante o sono (Gonzales et al., 2020). Em contrapartida, outro estudo realizado com jovens, na cidade de Pelotas (Rio Grande do Sul), em 2011, não encontrou diferenças significativas entre os sexos na prevalência de sintomas de depressão, ansiedade e estresse 
(Lopez et al., 2020). De acordo com o esse autor, é necessário ter um olhar igualitário quando da avaliação dos estados emocionais dos indivíduos, independente do sexo.

É importante salientar que as mulheres, historicamente, tiveram menor acesso à escolaridade, foram mais conduzidas a assumir as tarefas domésticas e ainda são submetidas a ocupações de menor renda e prestígio, e menores salários (Marques et al., 2020). As mulheres são mais preocupadas com a saúde e mais atentas a seus sinais e sintomas, e os resultados do estudo mostram uma atenção maior à saúde mental e à qualidade do sono, um ponto importante também é a intensificação das rotinas diárias das mulheres, incluindo cuidados com as crianças e os idosos, a limpeza da casa e preparo de refeições, atividades que na maior parte das vezes recaem sobre elas, além do crescimento da violência doméstica, durante a pandemia e o contingente distanciamento social (Shaw et al., 2018).

No presente estudo, universitários mais jovens se mostrarem mais afetados no domínio psicológico da qualidade de vida e apresentaram níveis significativamente maiores de estresse, ansiedade e depressão (Tabela 5). Isso pode ser explicado pelo fato de que indivíduos mais jovens, enfrentando mudanças inerentes a transição da adolescência para a vida adulta, podem ser mais susceptíveis ao desenvolvimento de sintomas de estresse, ansiedade e depressão, bem como prejuízos na qualidade de vida (Oliveira et al., 2020). Além disso, o fechamento das universidades e a incerteza sobre a conclusão dos períodos escolares podem ter prejudicado a saúde mental desses indivíduos (Barroso et al., 2019).

Observou-se que os estudantes que estiveram em cidades do interior foram significativamente mais prejudicados no domínio físico da qualidade de vida (Tabela 6). Isso sugere que eles podem ter sentido menos disposição para realizar suas tarefas diárias e assistir as aulas remotas.A falta de um ambiente adequado e a necessidade de lidar com atividades domésticas, cônjuge, filhos e tantas outras atribuições pode ter refletido nessa visão de prejuízo do domínio físico (Shaw et al, 2018). Outra variável importante relacionada à saúde física e ao bem-estar das pessoas é a prática de atividades físicas.Com o contexto do isolamento social, espaços tipicamente destinados a prática de atividades físicas, como academias e parques públicos foram fechados, dificultando a prática de atividade física e prejudicando a saúde geral dos indivíduos (Pitanga et al., 2020) de isolamento social, podendo ocasionar fadiga, falta de energia e indisposição, impactando o domínio físico da qualidade de vida (Bezerra et al, 2020).

O isolamento social associado a solidão pode ter contribuído para o impacto nos domínios psicológico e social da qualidade de vida dos estudantes (Tabela 7). De fato, a literatura relatou que estudantes mais solitários são mais susceptíveis a problemas físicos e mentais, que podem inclusive repercutir negativamente no desempenho acadêmico (Bezerra et al, 2020). O prejuízo observado no domínio social também pode estar relacionado ao isolamento, uma vez que o apoio social pode ser considerado um fator de proteção que ajuda as pessoas a lidarem com situações estressantes de forma mais eficaz, aumentando até as respostas físicas do corpo diante de situações difíceis (Guimarães, 2014). Daí a importância de recorrer a mecanismos não presenciais para manter o contato com familiares e amigos, como ligações telefônicas e chamadas de vídeo (Pais-Ribeiro et al., 2001).

Houve correlação positiva entre estresse e as variáveis ansiedade e depressão; assim como entre depressão e ansiedade (Tabela 8). De fato, em uma situação de pandemia, a exacerbação de sinais e sintomas de desvios psiquiátricos e a falta de habilidade para lidar com situações de emergência são esperados (Maia \& Dias, 2020). Tais sintomas podem ser pontuais ou se estenderem após o término do isolamento social (Bezerra et al., 2020).

A correlação negativa entre sintomas de estresse, ansiedade e depressão com o domínio psicológico da qualidade de vida (Tabela 9), como também apresentado em outro estudo, indica a eficácia dos instrumentos de avaliação utilizados (WHOOL- breve e EADS) (Pais-Ribeiro et al., 2001).

O presente estudo apresentou como principais limitações o tamanho e a conveniência da amostra. Uma vez que o universo foi composto por estudantes de odontologia de uma única instituição de ensino superior da cidade de João Pessoa, os 
resultados não são representativos do comportamento dos universitários como um todo, mas apenas do universo pesquisado. Entretanto, vale salientar que se alcançou a amostra estimada no cálculo amostral (com nível de confiança de 95\% e margem de erro de 5\%), o que permite realizar uma extrapolação dos resultados da amostra para a população estudada.

Ainda assim, os achados foram extremamente relevantes e possibilitaram identificar que mulheres e estudantes mais jovens dessa população foram mais afetados em termos de qualidade de vida e acometidos por sintomas de ansiedade, estresse e depressão, como consequência da pandemia. Isso só foi possível porque associou-se dois instrumentos de mensuração eficazes (WHOOL- breve e EADS) e que apresentaram, inclusive, correlação entre as variáveis.

Diante desse quadro, sugere-se que estudos de abrangência nacional sejam realizados, visando identificar o impacto do isolamento social decorrente da pandemia no bem-estar de universitários, o que possibilitaria as instituições de ensino superior subsídios para ações de suporte aos seus estudantes.

\section{Conclusão}

Os resultados forneceram importantes indicadores a respeito da qualidade de vida e da presença de sintomas de estresse, ansiedade e depressão na população estudada.

A maior parte dos estudantes apresentaram níveis considerados normais de estresse, ansiedade e depressão, além de prejuízo nos domínios físico e psicológico da qualidade de vida. Mulheres e estudantes mais jovens apresentaram níveis significativamente maiores de estresse, ansiedade e depressão. Mulheres foram expressivamente mais prejudicadas nos domínios físico, psicológico e ambiental da qualidade de vida e estudantes mais jovens, no domínio psicológico.

Houve prejuízo significativo no domínio físico da qualidade de vida daqueles que estiveram em cidades do interior, prejuízo nos domínios psicológico e social daqueles que estiveram sozinhos durante o período de isolamento social. Houve ainda correlação positiva entre as variáveis estresse, ansiedade e depressão, correlação significativa negativa entre domínios de qualidade de vida e as variáveis estresse, ansiedade e depressão, sendo maior entre depressão e o domínio psicológico.

O distanciamento social alterou significativamente a rotina das pessoas e diminuiu o contato físico o que gerou repercussões na renda familiar e na saúde física e mental em todo o mundo. Tendo em vista os resultados apresentados é importante ressaltar a necessidade de medidas para o cuidado e prevenção de transtornos psicológicos. Estudos que possam investigar a melhor forma de intervir e auxiliar esses indivíduos se fazem necessários. Sugere-se a elaboração de estudos mais amplos para mensurar os impactos da pandemia na saúde mental dos indivíduos, como exemplo, a nível nacional ou regional, permitindo uma maior generalização dos dados e uma compreensão mais ampla dos fatores estudados na presente pesquisa. Além disso, serviços psicológicos voltados a população estudantil são fundamentais para evitar o desenvolvimento de transtornos mentais. Nesse sentido, escutas psicológicas, materiais instrutivos e monitoramento dos estudantes podem ser fundamentais.

\section{Referências}

Asmundson, G. J. G., \& Taylor, S. (2020). Coronaphobia: fear and the 2019-nCoV outbreak. Journal of Anxiety Disorders, 70, 102196. 10.1016/j.janxdis.2020.102196

Barros, M. B. de A., Lima, M. G., Malta, D. C., Szwarcwald, C. L., de Azevedo, R. C. S., Romero, D., Souza Júnior, P. R. B. de., Azevedo, L. O., Machado, I. E., Damacena, G. N., Gomes, C. S., Werneck, A. de O., de Pina, M. de F., \& Gracie. R. (2020) Relato de tristeza/depressão, nervosismo/ansiedade e problemas de sono na população adulta brasileira durante a pandemia de COVID-19. Epidemiol. Serv. Saúde, 29(4), 1-12. https://doi.org/10.1590/S167949742020000400018

Barroso, S, M., Oliveira, N. R., \& de. Andrade, V. S. (2019). Solidão e Depressão: Relações com Características Pessoais e Hábitos de Vida em Universitários, Psicologia: Teoria e Pesquisa, 35, e35427. https://dx.doi.org/10.1590/0102.3772e35427.

Bezerra, A. C. V., Silva, C. E. M., da Soares, F. R. G., \& Silva, J. A. M. (2020). Fatores associados ao comportamento da população durante o isolamento social na pandemia de COVID-19. Ciênc. saúde coletiva, 25(1), 2411-2421. https://www.cienciaesaudecoletiva.com.br/artigos/fatores-associados-aocomportamento-da-populacao-durante-o-isolamento-social-na-pandemia-de-covid19/17551?id=17551. 
Cardoso, R. F., Alberto, D. de S., Maués, S. C. C., da Silva, S. R. M., Abreu, A. da C., Coelho, J. dos S. M., Marinho, M. T. B., Pinto, I. C., \& Meireles, A. A. V. (2021). COVID-19: Um desafio epidemiológico. Research, Society and Development, 10(7), e32110716313. https://dx.doi.org/10.33448/rsd-v10i7.16313.

Carvalho, P. M. M., Moreira, M. M., \& Oliveira, M. N. A., Landim, J. M. M., \& Rolim Neto, M. L. (2020) The psychiatric impact of the novel corona virus outbreak. PsychiatryResearch, 286: 112902. 10.1016/j.psychres.2020.112902.

Cayetano, M. H., Tavarez, M. G. J., Araújo, M. E., Martins, J. S., Crozato, E. M., \& Carrer, F. C. A. (2019). O perfil dos estudantes de Odontologia é compatível com o mercado de trabalho no serviço público de saúde brasileiro? Revista da Abeno, 19 (2), 2-12. https://doi.org/10.30979/rev.abeno.v19i2.736.

Costa, S. de M., Durães, S. J. A., \& de Abreu, M. H. N. G. (2020). Feminização do curso de odontologia da Universidade Estadual de Montes Claros. Ciênc. saúde coletiva, 15(1), 1865-1873. https://doi.org/10.1590/S1413-81232010000700100.

Delben-Barros, P., Cruz, R. M., Trevisan, K. R. R., Gai, M. J. P., Carvalho, R. V. C., Carlotto, P. A. C., Alves, R. B., Silvestre, D., Renner, R. B., da Silva, A. G., \& Malloy-Diniz, L. F. (2020). Saúde Mental em Situação de Emergência: Covid-19. Rev. Debates em Psychiatry, 10(22), 18-28. https://d494f813-3c95463a-898c-ea1519530871.filesusr.com/ugd/c37608_909ea3ff3b1c4ad3a032a853f68315b7.pdf.

Fleck, M. P., d Leal, A. O. F., Louzada, S., Xavier, M., Chachamovich, E., Vieira, G., dos Santos, L., \& Pinzon, V. Desenvolvimento da versão em português do instrumento de avaliação de qualidade de vida da OMS (WHOQOL-100). Rev. Bras. Psiquiatr, 21 (1), 19-28. https://doi.org/10.1590/S151644461999000100006

Gonçalves, A. M. C., Teixeira, M. T. B., Gama, J. R. de A., Lopes, C. S., Silva, G. A., Gamarra, C. J., \& Duque, K. de C. D. (2018) Prevalência de depressão e fatores associados em mulheres atendidas pela Estratégia de Saúde da Família. J. bras. psiquiatr, 67(2), 101-109. https://doi.org/10.1590/00472085000000192.

Gonzales, B. M., Silva, W. R., da Maroco, J., \& Campos, J. A. D. B (2019). Escala de Depressão, Ansiedade e Estresse: propriedades psicométricas e prevalência das afetividades. J. bras. psiquiatr, 68 (1), 32-41. https://doi.org/10.1590/0047-2085000000222.

Guimarães, M. F. Depressão ansiedade, estresse e qualidade de vida de estudantes de universidades pública e privada. Dissertação, curso de mestrado. Universidade Metodista de São Paulo. SP. 1-96. 2014. http://tede.metodista.br/jspui/handle/tede/1348.

Guo, J. X., Xie, H., Liang, M., \& Wu, H. (2020). COVID-19: a novel coronavirus and a novel challenge for oral healthcare. Clin Oral Investig, 7, 1-2. $10.1007 / \mathrm{s} 00784-020-03291-8$

Hossain, M. M., Sultana, A., \& Purohit, N. (2020). Mental health outcomes of quarantine and isolation for infection prevention: a systematic umbrella review of the global evidence. PsyArXi, 42: e2020038. https://doi.org/10.4178/epih.e2020038.

Iyer, P., Aziz, K., \& Ojcius, D. M. (2020). Impact of COVID-19 on dental education in the United States. J Dent Educ, 84(6), 718-722. 10.1002/jdd.12163.

Lopez, M. R. A., Ribeiro, J. P., Ores, L. da C., Jasen, K., Souza, L. D. de M., Pinheiro, R. T., \& da Silva R. A. (2020). Depressão e qualidade de vida em jovens de 18 a 24 anos no sul do Brasil. Rev Psiquiatr, 3 (2), 103-108. https://www.scielo.br/j/rprs/a/HjPQnyGq9c4p8qsMxWCTnrS/?format=pdf\&lang=pt.

Malloy-Diniz, L. F., Costa, D. de S., Loureiro, F., Moreira, L., Silveira, B. K. S., de Sadi, H. M., Apolinario-souza, T., Alvim-Soares, A., Nicolato, R., de Paula, J. J., Miranda, D., Pinheiro, M. I. C., Cruz, R. M., \& Silva, A. G. (2020). Saúde mental na pandemia de COVID-19: considerações práticas multidisciplinares sobre cognição, emoção e comportamento. Debates em psiquiatria, 10, 46-68. https://d494f813-3c95-463a-898cea1519530871.filesusr.com/ugd/c37608_59b07f09964c41cc8bd976bc1cc0e402.pdf.

Maia, B. R., \& Dias, P. C. (2020). Ansiedade, depressão e estresse em estudantes universitários: o impacto da COVID-19. Estudos de Psicologia, 37 , e200067. https://doi.org/10.1590/1982-0275202037e200067.

Marques, E. S., Hasselmann, M. M., Reichenhein, M. E., Moraes, C. L., \& Deslandes, S. F. (2020). A violência contra mulheres, crianças e adolescentes em tempos de pandemia pela COVID-19: panorama, motivações e formas de enfrentamento. Cad. Saúde Pública, 34(4) e00074420. 10.1590/0102311 X00074420.

Moreira, C. de L., \& Tonon, T. C. A. (2021) Desafios de estudantes concluintes do curso de bacharelado em enfermagem, diante do estágio supervisionado e a pandemia da Covid-19. Research, Society and Development, 10(7), e25710716640. https://rsdjournal.org/index.php/rsd/article/view/16640/14761.

Oliveira, W. A., da Silva, J. L., Andrade, A. L. M., de Micheli, D., Carlos, M. D., \& Silva, M. A. L. (2020). A saúde do adolescente em tempos da COVID-19: scopingreview, 36 (8), 1-14. https://doi.org/10.1590/0102-311X00150020.

Oxfam Brasil. (2018). País Estagnado: um retrato das desigualdades brasileiras, Retrieved Jun 29, 2021, from https://sinapse.gife.org.br/download/paisestagnado-um-retrato-das-desigualdades-brasileiras

Pais-Ribeiro, J. L., Horonado, A., \& Leal, I (2001). Contribuição para o estudo da adaptação portuguesa das Escalas de Ansiedade Depressão e Stress de Lovibond e Lovibon. Psicologia Saúde \& Doenças, 36(2), 229-239. https://www.redalyc.org/pdf/362/36250207.pdf.

Pitanga, J. G., Beck, C. C., \& Pitanga, C. P. S. (2020). Atividade Física e Redução do Comportamento Sedentário durante a Pandemia do Coronavírus. Ponto de Vista. Arq. Bras, 1-3. https://doi.org/10.36660/abc.2020023.

Poulsen, M. N., Bailey-Davis, L., Pollak, J., Hirsch, A. G., \& Schwartz, B. S. (2019) Household food insecurity and home food availability in relation to youth diet, body mass index, and adiposity. J AcadNutr Diet, 119(10), 1666-1675. 10.1016/j.jand.2019.01.001.

Spagnuolo, G., De Vito, D., \& Rengo S. T. (2020). COVID-19 Outbreak: An Overview on Dentistry. Int. J. Environ. Res. Public Health, 17 (6), 2094. 10.3390/ijerph17062094.

Sabino-silva, R., Jardim, A. C. G. J. M., \& Siqueira, W. L. (2020). Coronavirus COVID-19 impacts to dentistry and potential salivary diagnosis. Clinical Oral Investigations, 24, 1619-1621. 10.1007/s00784-020-03248. 
Research, Society and Development, v. 10, n. 8, e34710817481, 2021

(CC BY 4.0) | ISSN 2525-3409 | DOI: http://dx.doi.org/10.33448/rsd-v10i8.17481

Schmidt, B., Crepaldi, M. A., Bolze, S. D. A., Neiva-Silva, L., \& Demenech, L. M. (2020). Saúde mental e intervenções psicológicas diante da pandemia do novo coronavírus (COVID-19). Estudos de Psicologia, 37, e200063. https://doi.org/10.1590/1982-0275202037e200063.

Shaw, S., Wherton, J., Vijayaraghavan, S., Morris, J., Bhattacharya, S., Hanson, P., Campbel-Richards, D., Ramoutar, S., Collard, A., Hodkinson, I., \& Greenhalgh, T. (2018). Advantages and limitations of virtual online consultations in a NHS acutetrust: the VOCAL mixed-methods study. NIHR Journals Library, 6(21), 10.3310/hsdr06210.

Shojaei, S. F., \& Masoumi, R. (2020). The importance of mental health training for psychologists in COVID-19 outbreak. Middle East Journal of Rehabilitation and Health Studies, 7 (2), e102846. 10.5812/mejrh.102846.

Wang, C., Pan, R., Wan, X., Tan, Y., Xu, L., Ho, C. S., \& Ho, R. C. (2020). Immediate Psychological Responses and Associated Factors during the Initial Stage of the 2019 Coronavirus Disease (COVID-19) Epidemic among the General Population in China. Int J Environ Res Public Health, 6;17 (5), 1729. 10.3390/ijerph17051729. 